Cahiers

de la Recherche Fondamentaux
Cahiers de la recherche sur les droits fondamentaux

$17 \mid 2019$

La motivation des actes administratifs. Le droit français à la lumière du droit administratif comparé

\title{
La motivation des actes administratifs en droit espagnol : vers une réflexion sur les rapports entre «fond » et « forme »
}

Rhita Bousta

\section{OpenEdition}

\section{Journals}

Édition électronique

URL : https://journals.openedition.org/crdf/3725

DOI : $10.4000 /$ crdf. 3725

ISSN : 2264-1246

Éditeur

Presses universitaires de Caen

Édition imprimée

Date de publication : 31 décembre 2019

Pagination : 77-85

ISBN : 978-2-84133-960-0

ISSN : $1634-8842$

\section{Référence électronique}

Rhita Bousta, «La motivation des actes administratifs en droit espagnol : vers une réflexion sur les rapports entre « fond » et « forme » », Cahiers de la recherche sur les droits fondamentaux [En ligne], 17 | 2019, mis en ligne le 06 février 2021, consulté le 15 novembre 2022. URL : http://

journals.openedition.org/crdf/3725; DOI : https://doi.org/10.4000/crdf.3725 


\title{
La motivation des actes administratifs en droit espagnol: vers une réflexion sur les rapports entre "fond " et «forme »
}

\author{
Rhita BOUSTA \\ Maîtresse de conférences en droit public à l'université de Lille \\ Professeure à l'université ouverte de Catalogne (Espagne)
}

I. Le caractère casuistique du devoir de motivation

A. La consécration légale du devoir de motivation: une liste de cas

B. La modulation prétorienne du devoir de motivation

II. Vers une approche substantielle de la motivation

A. Le rattachement du devoir de motivation à des principes et droits constitutionnels

B. Un ordre juridique contribuant à la réflexion sur les rapports entre le fond et la forme

«Si l'on ne peut s'entendre au préalable sur les solutions précises à apporter aux problèmes, du moins peut-on s'entendre sur les procédures $[\ldots] »^{1}$. Au cœur du droit administratif européen ${ }^{2}$, la motivation fait ainsi l'objet d'un certain consensus. Figurant à l'article 41 de la Charte des droits fondamentaux de l'Union européenne relatif au droit à une bonne administration ${ }^{3}$, le devoir de motivation est invocable par «toute personne » ${ }^{4}$, même si son contenu pourra donner lieu à diverses interprétations ${ }^{5}$. En réalité, cet article ne fait que confirmer une jurisprudence bien établie ${ }^{6}$. Ainsi, bien que particulièrement réceptif à ce nouveau droit ${ }^{7}$, le juge espagnol l'évoque uniquement

1. N. Luhmann, La légitimation par la procédure, L. K. Sosoe, S. Bouchard (trad.), Laval, Les Presses de l'université Laval (Dikè), 20o1, p. 144. L'auteur critique cette approche jugée simpliste, qui marque la «perte du droit naturel par le droit procédural» (p. 144).

2. J.-B. Auby, J. Dutheil de la Rochère, "À propos de la notion de droit administratif européen ", Revue française d'administration publique, ${ }^{\circ}{ }^{123}$, 2007, Varia, p. 375-385; R. Alonso Garcia, Derecho comunitario y derechos nacionales. Autonomía, integración e interacción, Buenos Aires, Abeledo Perrot, 1999, p. 19-43.

3. J. Ziller, «Droit à une bonne administration», Jurisclasseur Libertés, fasc. 1040, 10 septembre 2007.

4. Sur l'extension de l'invocabilité du fait de l'inscription à l'article 41 de la Charte des droits fondamentaux de l'Union européenne, voir J.-L. Autin, C. Ribot, Droit administratif général, 5 éd., Paris, Litec (Objectif droit), 2007, p. 387-388; K. Michelet, «La Charte des droits fondamentaux de l'Union européenne et la procédure administrative non contentieuse », L'actualité juridique. Droit administratif, no 14, 14 octobre 2002, p. 949-955.

5. A. Pêcheul, «La Charte des droits fondamentaux de l'Union européenne», Revue française de droit administratif, $\mathrm{n}^{\circ} 3$, mai-juin 2001 , p. 693.

6. CJCE, GC, 10 juillet 2008, Bertelsmann et Sony Corporation of America c. Impala, C-413/o6 P, non publié, concl. de l'avocat général J. Kokott, présentées le 13 décembre 2007, point 97; L. Burgorgue-Larsen, "La "force de l'évocation" ou le fabuleux destin de la Charte des droits fondamentaux de l'Union européenne", in Mélanges en l'honneur de Pierre Pactet. L'esprit des institutions, l'équilibre des pouvoirs, Paris, Dalloz, 2003, p. 92-95.

7. Le Tribunal constitutionnel y faisait référence avant même l'entrée en vigueur de cette charte: STC, Ass., $\mathrm{n}^{\circ}$ 292/2000, 30 novembre 2000 , Boletín oficial del Estado, $\mathrm{n}^{\circ}$ 4, 4 janvier 2001, p. 104. Les abréviations utilisées dans les références aux arrêts sont les suivantes: FJ (fondamento jurídico), STC (sentencia del Tribunal constitucional), STS (sentencia del Tribunal supremo), STSJ (sentencia del Tribunal superior de justicia). 
comme fondement supplémentaire, voire accessoire du devoir de motivation ${ }^{8}$.

On pourrait entrevoir ses prémices dans la loi portant sur les bases de la procédure administrative espagnole de $1889^{9}$. Mais c'est surtout la loi relative à la procédure administrative de $1958^{10}$ qui consacre expressément un tel devoir. Adoptée en pleine période franquiste, cette réforme est l'œuvre de la doctrine espagnole appelant à la fois à une unification et à un renouveau de la procédure administrative alors conçue comme une méthode d'action de l'administration indépendamment de la politique. Violant par ailleurs des droits fondamentaux, le régime franquiste a ainsi concédé certains droits procéduraux. Adoptée sans difficultés, cette loi avait même fait l'objet de propagandes vantant la rapidité et l'efficacité de l'administration franquiste $^{11}$. Sans surprises, le champ d'application de la motivation fut réduit à une liste de cas : les actes limitant les droits subjectifs, résolvant des recours administratifs, devant être motivés selon une loi spéciale, se démarquant de précédents ou d'avis d'organes consultatifs ainsi que les accords relatifs à la suspension d'un acte ayant fait l'objet d'un recours ${ }^{12}$.

Le passage à un «État de droit social et démocratique ${ }^{13}$, concept inspiré du droit allemand ${ }^{14}$, eut un impact non négligeable sur le droit administratif espagnol tout comme l'entrée de la Péninsule ibérique dans la Communauté européenne ${ }^{15}$. Ainsi, contrairement à son homologue français, le législateur national espagnol a, de longue date, codifié les règles et les principes régissant le fonctionnement administratif à travers la fameuse loi de 1992 relative au régime juridique des administrations publiques et à la procédure administrative de droit commun (Ley de Régimen Jurídico de las Administraciones Públicas y del Procedimiento Administrativo Común) ${ }^{16}$. Modifié par une loi datant de 1999, son article 54 relatif au devoir de motivation réitère la structure de la disposition de la loi de 1958 en ouvrant toutefois son champ d'application à de nouveaux cas. Cette consécration sous forme de liste - d'ailleurs commune au droit français ${ }^{17}$ - n'a pas été remise en cause par la récente réforme de $2015^{18}$. Le champ d'application a simplement été de nouveau élargi.

La jurisprudence espagnole tend, quant à elle, à généraliser le devoir de motivation en se fondant sur l'interdiction de l'arbitraire ${ }^{19}$. C'est ainsi que certains cas absents de la liste législative s'y trouvent néanmoins soumis. Toutefois, comme nous le verrons par la suite, le juge se garde encore de censurer certains actes administratifs non motivés si bien qu'il apparaît encore risqué d'ériger la motivation en principe général du droit, même si les actes non soumis à ce devoir sont conçus, pour la majeure partie de la doctrine ${ }^{20}$, comme des exceptions à une règle générale implicite.

À titre liminaire, on s'accordera à définir la motivation comme l'extériorisation formelle des motifs de l'acte. En Espagne, le terme motivación (motivation) est alors distingué de celui de fundamentación (motif). Rappelons également que le motif se différencie de l'objectif ${ }^{21}$ en ce que le premier relève de la qualification des faits extérieurs et préexistants à l'acte. Enfin, tout comme en France, la motivation est souvent appréhendée de manière objective et non comme un droit subjectif. Celle-ci permet avant tout de renforcer les principes d' " objectivité et de transparence de l'action administrative $»^{22}$.

8. Pour exemples: STS, $5^{\mathrm{e}}$ sect., $\mathrm{n}^{\mathrm{o}}$ 1841/2005, 23 mars $2005, \mathrm{FJ} 4$; STS, $5^{\mathrm{e}}$ sect., $\mathrm{n}^{\circ} 6089 / 2006,27$ juin $2004, \mathrm{FJ}_{3}$; STS, $3^{\mathrm{e}}$ sect, $\mathrm{n}^{\circ} 2156 / 2004,29$ mars 2004 , FJ4; STSJ de la Communauté de Valence, nº 407/2002, 18 novembre 2005, FJ1.

9. Ley de Bases del Procedimiento Administrativo, 19 octobre 1889, Gaceta de Madrid, nº 298, 25 octobre 1889, t. IV, p. 237. Cette loi n’introduit pas expressément de devoir de motivation, mais créé pour la première fois un droit de l'intéressé et du public à être entendu (art. 2, $\$ 10$ ), ce qui impliquerait à notre sens, en amont, un minimum d'explication de la décision de la part de l'administration.

10. Ley de Procedimiento Administrativo, 17 juillet 1958, Boletín oficial del Estado, n 171,18 juillet 1958, p. 1275.

11. Pour un rappel récent sur ce point précis: C. Ceccarini, El órgano instructor del procedimiento administrativo en España y el responsable del procedimiento administrativo en Italia: un estudio comparado, thèse de doctorat, Universidad Complutense de Madrid, 2017 , p. 54, note 92.

12. Ley de Bases del Procedimiento Administrativo, art. 43 .

13. Art. $1^{\text {er }}$ de la Constitution espagnole: «Estado social y democrático de Derecho ». Toutes les traductions de l'espagnol au français ont été faites par l'auteure de cet article.

14. L. Parejo Alfonso, Estado social y administración pública. Los postulados constitucionales de la reforma administrativa, Madrid, Civitas (Monografías), 1983, p. 29-95.

15. S. Muñoz Machado, Tratado de derecho administrativo y derecho público general, Madrid, Thomson Civitas, 2004, t. I, p. 48-67.

16. Loi no 3o/1992 du 26 novembre 1992, Ley de Régimen Jurídico de las Administraciones Públicas y del Procedimiento Administrativo Común, Boletín oficial del Estado, $\mathrm{n}^{\circ} 285,27$ novembre 1992, p. 40.300 (modifiée par la loi n 4/1999 du 13 janvier 1999, Boletín oficial del Estado, n 12 , 14 janvier 1999).

17. Art. 211-2 du Code des relations entre le public et l'administration, $2^{\mathrm{e}}$ éd., Paris, Dalloz, 2018, p. 81.

18. Loi n ${ }^{\circ}$ 39/2015 du $1^{\text {er }}$ octobre 2015, Ley del Procedimiento Administrativo Común de las Administraciones Públicas, art. 35, Boletín oficial del Estado, $\mathrm{n}^{\circ}$ 236, 2 octobre 2015, p. 89343. Pour un regard critique sur cette réforme, voir J. A. Santamaría Pastor, «Los proyectos de Ley del Procedimiento Administrativo Común de las Administraciones Públicas y de Régimen Jurídico del Sector Público: una primera evaluación», Documentación administrativa, $\mathrm{n}^{\circ}$ 2, janvier-décembre 2015, en ligne: https://revistasonline.inap.es/index.php/DA/article/view/10258/10754; pour un commentaire exhaustif de cette loi, voir Comentarios a la Ley 39/2015 de Procedimiento Administrativo Común de las Administraciones Públicas, C. Campos Acuña (dir.), Madrid, Wolters Kluwer, 2017 (s'agissant plus précisément du devoir de motivation: p. 297-300).

19. Pour une étude récente et complète en la matière, voir R. M. Navarro González, La motivación de los actos administrativos, Madrid, Aranzadi, 2017.

20. M. M. Fernando Pablo, La motivación del acto administrativo, Madrid, Tecnos, 1993, p. 236; J. Gonzalez Perez, F. Gonzalez Navarro, Comentarios a la Ley de Régimen Jurídicos de las Administraciones Públicas y del Procedimiento Administrativo Común (Ley 30/1992, de 26 de Noviembre), $2^{\mathrm{e}}$ éd., Madrid, Civitas, 1999, vol. I, p. 1484.

21. R. Bonnard, Précis de droit administratif, $4^{\mathrm{e}}$ éd., Paris, LGDJ, 1943, p. 28-29.

22. STS, $3^{\mathrm{e}}$ sect., $\mathrm{n}^{\circ}$ 1091/2005, 22 février 2005, FJ2 (le juge reprend ici l'exposé des motifs d'un décret royal datant de 1994); J. Rodríguez-Arana, Derecho administrativo español, t. II, Acto administrativo, procedimiento administrativo y revisión de la actuación administrativa, El Ferrol, Netbiblo, 2009, p. 27. 
La motivation est alors indéniablement liée au pouvoir discrétionnaire ${ }^{23}$ de l'administration. Souvent conçu comme résiduel, celui-ci est alors entendu comme ce qui n'est pas de compétence liée ${ }^{24}$. En d'autres termes, son étendue dépendrait du degré d'indétermination de la loi, voire de la Constitution, qui est envisagée en Espagne comme la première source de «discrétionnalité» de l'administration ${ }^{25}$. Selon une conception répandue dans cet ordre juridique ${ }^{26}$, la norme ne constitue pas uniquement une limite au pouvoir discrétionnaire; elle en est le fondement ${ }^{27}$ et appelle à son déploiement ${ }^{28}$. Aussi la doctrine ${ }^{29}$ affirmet-elle que les principes figurant à l'article 103 de la Constitution espagnole (soumission à l'intérêt général, respect de la loi et du droit ainsi que des principes d'efficacité, de hiérarchie, de décentralisation, de déconcentration et de coordination) invitent l'administration à choisir la «meilleure voie possible $»^{30}$ en usant de son pouvoir discrétionnaire. La soumission constitutionnelle de l'administration à l'intérêt général dicte cette dernière dans le choix de cette solution optimale ${ }^{31}$. Cette approche se retrouve, en France, sous la plume de certains auteurs ${ }^{32}$ qui, prenant le contre-pied des définitions négatives empruntes d'un certain légicentrisme, envisagent le pouvoir discrétionnaire comme «l'adaptation au réel de la règle de droit $»^{33}$. À cette volonté de "positivation» de la définition du pouvoir discrétionnaire s'ajoute la contestation de son antonymie avec la compétence liée ${ }^{34}$. En tous les cas, les règles de compétences, de motif ainsi que le but d'intérêt public encadrent ou du moins "accompagnent» ce pouvoir ${ }^{35}$.

En est-il de même de la motivation? Le caractère fondamentalement casuistique de ce devoir a donné lieu à une jurisprudence riche d'enseignements (I). Loin d'être systématiquement reléguée au rang des exigences de «pure forme», la motivation pourrait ainsi faire l'objet d'une approche substantielle (II).

\section{Le caractère casuistique du devoir de motivation}

À titre liminaire, signalons que l'article 35 de la loi relative à la procédure commune aux administrations publiques définit la motivation comme l'énoncé «succinct des faits et fondements juridiques ${ }^{36}$ de l'acte administratif. Cette formulation évasive, qui date de 1958, rappelle celle de l'article 3 de la loi française de $1979^{37}$ désormais codifié à l'article L. 211-5 du Code des relations entre le public et l'administration ${ }^{38}$ selon lequel la motivation «doit être écrite et comporter l'énoncé des considérations de droit et de fait qui constituent le fondement de la décision».

Selon la jurisprudence espagnole, la motivation peut être brève, voire "concise ${ }^{39}$, mais doit en tous les cas être suffisante. Qu'entendre alors par motivation «suffisante» ? Sous la plume du juge, celle-ci doit être « concrète ${ }^{40}$ en ce que l'administration doit mentionner les critères d'application de la règle de droit au cas particulier. Sont ainsi censurées les «références imprécises et génériques relatives aux considérations qui ont fondé la décision administrative $»^{41}$. Le juge exige traditionnellement l'énoncé des faits pertinents («fijación de los hechos determinantes»), leur confrontation à la norme ( «subsunción en la norma») ainsi qu'une mention succincte des raisons pour lesquelles la décision s'avère adéquate ("una especificación sucinta de las razones por las que [...] resulte adecuada la resolución ${ }^{42}$ ).

23. Selon un auteur, l'usage de l'expression «pouvoir discrétionnaire» remonterait aux écrits de Hans Hüber, «Niedergang des Rechts und Krise des Rechtsstaat», in Festgabe für Z. Giacometti, Zürich, Polygraphischer Verlag AG, 1953, p. 66; source: E. García de Enterría, La lucha contra las inmunidades del poder en el derecho administrativo (poderes discrecionales, poderes de gobierno, poderes normativos), $3^{\mathrm{e}}$ éd., Madrid, Civitas (Cuadernos), 1983, p. 24. Néanmoins, il semble difficile de cerner avec exactitude la genèse de cette expression, tant son existence est liée à la naissance même de l'administration publique.

24. L. Michoud, «Étude sur le pouvoir discrétionnaire de l'administration», Revue générale d'administration, t. III, 1914, p. 5-26; p. 193-214.

25. E. Desdentado Daroca, Discrecionalidad administrativa y planeamiento urbanístico. Construcción teórica y análisis jurisprudencial, $2^{\mathrm{e}}$ éd., Pamplune, Aranzadi (Monografías), 1999, p. 57; M. J. Alonso Mas, La solución justa en las resoluciones administrativas, Valence, Tirant Lo Blanch (Propuestas-Monografía), 1998, p. 236.

26. Contra : F. Garrido Falla, Tratado de derecho administrativo, $12^{\mathrm{e}}$ éd., Madrid, Tecnos, 1994, t. I, p. 184.

27. J. Tornos Mas, «La situación actual del proceso contencioso-administrativo », Revista de administración pública, nº 122, mai-août 1990, p. 111.

28. E. Desdentado Daroca, Discrecionalidad administrativa..., p. 70-71.

29. C. Chinchilla Marín, La desviación de poder, Madrid, Civitas (Monografías), 1989, p. 151; M. Beltrán de Felipe, Discrecionalidad administrativa y Constitución, Madrid, Tecnos (Temas clave de la Constitución española), 1995, p. 165-200; M. Sánchez Morón, Discrecionalidad administrativa y control judicial, Madrid, Tecnos (Ciencias jurídicas), 1994, p. 103-112; L. Parejo Alfonso, Crisis y renovación en el derecho público, Madrid, Centro de estudios constitucionales (Cuadernos y debates; 30), 1991, p. 73 sq.; F. Sainz Moreno, "Reducción de la discrecionalidad: el interés público como concepto jurídico", Revista española de derecho administrativo, no 8, janvier-mars 1976, p. 63-93.

30. C. Chinchilla Marín, La desviación de poder, p. 151.

31. Voir M. J. Alonso Mas, La solución justa..., p. 242; sur les concepts juridiques indéterminés: p. 274-320.

32. J.-C. Venezia, Le pouvoir discrétionnaire, Paris, R. Pichon et R. Durand-Auzias, 1959, p. 105; p. $112-118$.

33. Ibid., p. 156

34. G. Timsit, «Compétence liée et principe de légalité », Recueil Dalloz, 1964, chronique, n 31, p. 219.

35. G. Vedel, P. Delvolvé, Droit administratif, $12^{\mathrm{e}}$ éd., Paris, PUF (Thémis. Droit public), 1992, t. II, p. 528-536.

36. Art. 35.1 de la loi $\mathrm{n}^{\mathrm{o}} 39 / 2015$ du $1^{\mathrm{er}}$ octobre 2015: «sucinta referencia de hechos y fundamentos de derecho ".

37. Loi $\mathrm{n}^{\mathrm{o}} 79-587 \mathrm{du} 11$ juillet 1979 relative à la motivation des actes administratifs et à l'amélioration des relations entre l'administration et le public, Journal officiel de la République française, 12 juillet 1979, p. 1711.

38. Code des relations entre le public et l'administration, p. 104.

39. STS, $5^{\mathrm{e}}$ sect., $\mathrm{n}^{\circ} 8090 / 1999,15$ décembre $1999, \mathrm{FJ} 2$ : «escueta ».

40. STS, $5^{\mathrm{e}}$ sect., $\mathrm{n}^{\mathrm{o}} 5312 / 2008,23$ septembre $2008, \mathrm{FJ} 4$.

41. STS, $5^{\mathrm{e}}$ sect., $\mathrm{n}^{\circ}$ 4313/2010, 9 juillet $2010, \mathrm{FJ}_{4}$ : « referencias imprecisas y genéricas sobre las consideraciones que han determinado".

42. STS, $6^{\mathrm{e}}$ sect., $\mathrm{n}^{\mathrm{o}} 1623 / 2003,10$ mars $2003, \mathrm{FJ}_{2}$. 
À notre sens, l'exemple espagnol illustre parfaitement le caractère casuistique du devoir de motivation des actes administratifs. À l'instar du droit français, la loi espagnole procède par l'énoncé d'hypothèses soumises à ce devoir (A). Il appartient alors au juge de moduler ce dernier selon le cas concret (B).

\section{A. La consécration légale du devoir de motivation: une liste de cas}

Premier constat: aucun principe général de motivation ne figure dans le texte de loi. Comme évoqué précédemment, l'article dispose, en premier lieu, que celle-ci consiste en l'énoncé «succinct des faits et fondements juridiques » ${ }^{43}$. La disposition espagnole énumère, en second lieu, les actes administratifs soumis à ce devoir.

Conservant à la fois cette brève définition et cette structure, la réforme de 2015 se contente d'introduire de nouveaux cas à la liste existante. Ses rédacteurs se sont ainsi gardés de consacrer un principe susceptible de s'appliquer à tout acte administratif.

Par ailleurs, cette réforme maintient une hypothèse faisant toujours l'objet d'un paragraphe distinct: les concours et autres procédures compétitives de sélection. L'actuel article 35.2 reproduit littéralement l'ancienne disposition (article 54.2) en renvoyant la question de la motivation aux dispositions spéciales et sectorielles. Dans un souci d'économie de procédure, et eu égard à l'éventuelle multiplicité de destinataires, la motivation de l'acte administratif n'est donc pas envisagée de manière générale. En revanche, l'existence de motifs fondant la décision - même en l'absence de motivation - reste une exigence s'imposant dans tous les cas ${ }^{44}$.

En dehors de cette hypothèse, l'ancienne disposition résultant de la loi précitée de 1992, telle que modifiée en 1999, prévoyait dix cas soumis au devoir de motivation ${ }^{45}$ : les actes administratifs portant atteinte à des droits subjectifs ou à des intérêts légitimes; ceux mettant fin à un recours administratifs ou à toute réclamation précédant les procédures contentieuse et d'arbitrage; la révision, initiée par l'administration, de dispositions ou d'actes administratifs; les actes se démarquant de critères émis par de précédents actes ou par des organes consultatifs; ceux découlant de l'exercice d'un pouvoir discrétionnaire; les accords consistant en la suspension d'un acte, la prolongation d'un délai ou la mise en œuvre d'une procédure d'urgence; les mesures provisoires; et enfin, les actes soumis au devoir de motivation selon une loi ou un règlement spéciaux.

Inspirée notamment d'une ancienne résolution du Conseil de l'Europe ${ }^{46}$, la première hypothèse (actes administratifs portant atteinte à des droits subjectifs ou à des intérêts légitimes) n'est pas surprenante. En résumé, une motivation s'impose en cas de décision administrative défavorable ou de décision favorable mais portant préjudice à un tiers. En revanche, si la décision favorable ne limite pas les droits ou les intérêts d'un tiers, celle-ci ne doit pas nécessairement être motivée selon le texte de loi.

L'hypothèse relative au pouvoir discrétionnaire - sur laquelle nous reviendrons par la suite - soulève quant à elle un intérêt certain. À ce sujet, le Tribunal suprême précisait, dès 1999, que le pouvoir discrétionnaire conféré à l'administration implique le respect, par cette dernière, d'une certaine " "raisonnabilité" dans un contexte socioculturel déterminé» ${ }^{47}$.

Enfin, l'hypothèse se référant aux «précédents » administratifs semble intéressante d'un point de vue comparatif. Inspirée de la notion anglo-saxonne de precedent, le précédent administratif peut être défini comme la «réponse ou la décision adoptée antérieurement par une même administration pour un cas ou une série de cas similaires à ceux en cause ${ }^{48}$. Ici, le devoir de motivation met en exergue la nécessité de cohérence de l'action administrative et de respect du principe de confiance légitime. La notion de précédent administratif a donc été invoquée et examinée par le juge dès l'édiction de cette disposition, dans de nombreuses hypothèses, telles que le contentieux de la rénovation des concessions du domaine publique ${ }^{49}$.

La réforme de 2015 a introduit d'autres cas désormais expressément soumis au devoir de motivation ${ }^{50}:$ les actes rejetant pour irrecevabilité les demandes de révision, de

43. Art. 35.1 de la loi $n^{\circ} 39 / 2015$ du $1^{\text {er }}$ octobre 2015 .

44. Art. 35.2 de la loi $\mathrm{n}^{\mathrm{o}} 39 / 2015$ du $1^{\mathrm{er}}$ octobre 2015: «La motivación de los actos que pongan fin a los procedimientos selectivos y de concurrencia competitiva se realizará de conformidad con lo que dispongan las normas que regulen sus convocatorias, debiendo, en todo caso, quedar acreditados en el procedimiento los fundamentos de la resolución que se adopte».

45. Art. 54.1 de la loi nº 30/1992 du 26 novembre 1992: «Serán motivados, con sucinta referencia de hechos y fundamentos de derecho: a) Los actos que limiten derechos subjetivos o intereses legítimos. b) Los que resuelvan procedimientos de revisión de oficio de disposiciones o actos administrativos, recursos administrativos, reclamaciones previas a la vía judicial y procedimientos de arbitraje. c) Los que se separen del criterio seguido en actuaciones precedentes o del dictamen de órganos consultivos. d) Los acuerdos de suspensión de actos, cualquiera que sea el motivo de ésta, así como la adopción de medidas provisionales previstas en los artículos 72 y 136 de esta Ley. e) Los acuerdos de aplicación de la tramitación de urgencia o de ampliación de plazos. f) Los que se dicten en el ejercicio de potestades discrecionales, así como los que deban serlo en virtud de disposición legal o reglamentaria expresa».

46. Comité des ministres du Conseil de l'Europe, résolution sur la protection de l'individu au regard des actes de l'administration, $\mathrm{n}^{\circ} 77 / 31$, 28 septembre 1977

47. STS, $7^{\mathrm{e}}$ sect., $\mathrm{n}^{\circ} 3829 / 1999,1^{\mathrm{er}}$ juin 1999, FJ5: «"Razonabilidad" en un marco socio-cultural determinado ».

48. M. Sánchez Morón, Derecho administrativo: parte general, $12^{\mathrm{e}}$ éd., Madrid, Tecnos, 2016, p. 94: «Respuesta o decisión adoptada con anterioridad por una misma Administración en un supuesto o enuna serie de supuestos similaires a aquel con el que se compara».

49. Pour exemple: STS, $3^{\mathrm{e}}$ sect., $\mathrm{n}^{\circ}$ 1261/1999, 24 février 1999, FJ2.

50. Art. 35.1 de la loi $\mathrm{n}^{\circ} 39 / 2015$ du $1^{\text {er }}$ octobre 2015: «Serán motivados, con sucinta referencia de hechos y fundamentos de derecho: a) Los actos que limiten derechos subjetivos o intereses legítimos. b) Los actos que resuelvan procedimientos de revisión de oficio de disposiciones o actos 
recours administratifs et d'arbitrage; les accords prévoyant la réalisation d'actes ou d'actions complémentaires à l'acte administratif principal; les actes rejetant des preuves avancées par les intéressés; ceux mettant fin à une procédure administrative en cas de force majeure; ceux constitutifs d'un désistement de l'administration suite à une procédure initiée de oficio.

Cet enrichissement de la liste d'hypothèses soumises au devoir de motivation soulève au moins une interrogation. En l'absence de critère clairement défini, assisterons-nous à une succession de réformes consistant à élargir cette liste au gré des hypothèses dégagées par la jurisprudence? L'hétérogénéité des cas mentionnés n'appelle-t-elle pas, à l'inverse, à une harmonisation de ce devoir sous forme de principe général? Dans ce contexte, et en l'absence d'un tel principe, le juge espagnol s'est arrogé un rôle important dans l'élaboration des contours du devoir de motivation.

\section{B. La modulation prétorienne du devoir de motivation}

Pour certains, la rédaction actuelle de l'article 35 de la loi relative à la procédure commune aux administrations publiques instaurerait un pouvoir prétorien contraire à la Constitution, qui limite le pouvoir du juge aux champs de compétence conférés par la loi ${ }^{51}$. Toutefois, la motivation d'un acte administratif n'est-elle pas, par nature, sujette à interprétations jurisprudentielles? Une rédaction du texte de loi davantage détaillée permettrait-elle réellement d'encadrer a priori la jurisprudence? Qu'il soit permis d'en douter. Il semble en effet que, quel que soit l'ordre juridique concerné, le contrôle juridictionnel du devoir de motivation est essentiellement modulable.
La «suffisance» de la motivation ne peut en effet être définie a priori, et son appréciation dépendra des circonstances du cas concret. De surcroît, celle-ci n'est pas systématiquement liée au degré de pouvoir discrétionnaire détenu par l'administration. En effet, l'exigence de motivation sera minime en cas de recrutement comportant une appréciation «subjective» de la part de l'administration (fort pouvoir discrétionnaire) mais pourra être tout aussi réduite en cas de compétence liée ou quasi liée (faible pouvoir discrétionnaire) si la décision administrative vise une multiplicité de destinataires, dans un souci d'économie procédurale.

Ici très poussée, la motivation fait, ailleurs, l'objet d'une autocensure de la part du juge. C'est le cas s'agissant des nominations aux emplois publics ${ }^{52}$ dont le contrôle est souvent réduit à l'interdiction de l'arbitraire ou au détournement de pouvoir. Traditionnellement, la jurisprudence espagnole envisage ces nominations comme « une composante spécifique et singulière de la catégorie générale des actes discrétionnaires». Cette singularité résiderait dans la «confiance [envers le candidat] que seule l'autorité administrative est à même d'apprécier ${ }^{33}$. En d'autres termes, le juge se refuse de contrôler «les raisons subjectives déterminant la confiance $»^{54}$ envers un candidat plutôt qu'un autre.

Cette liberté comporte toutefois des limites. C'est ainsi que, dans un arrêt de 2006 , le Tribunal suprême annula, pour défaut de motivation, la nomination de Don Salvador Francisco Javier Gómez Bermúdez au poste de président de la chambre pénale de l'Audiencia Nacional ${ }^{55}$. Le décret royal concerné omettait en effet le rapport de la commission de qualification, et, par-là, violait les «exigences constitutionnelles de mérite et de capacité» laissant présager une "empathie personnelle ou une affinité politique ${ }^{56}$. Cette jurisprudence renvoie

administrativos, recursos administrativos y procedimientos de arbitraje y los que declaren su inadmisión. c) Los actos que se separen del criterio seguido en actuaciones precedentes o del dictamen de órganos consultivos. d) Los acuerdos de suspensión de actos, cualquiera que sea el motivo de ésta, así como la adopción de medidas provisionales previstas en el artículo 56. e) Los acuerdos de aplicación de la tramitación de urgencia, de ampliación de plazos y de realización de actuaciones complementarias. f) Los actos que rechacen pruebas propuestas por los interesados. g) Los actos que acuerden la terminación del procedimiento por la imposibilidad material de continuarlo por causas sobrevenidas, así como los que acuerden el desistimiento por la Administración en procedimientos iniciados de oficio. h) Las propuestas de resolución en los procedimientos de carácter sancionador, así como los actos que resuelvan procedimientos de carácter sancionador o de responsabilidad patrimonial. i) Los actos que se dicten en el ejercicio de potestades discrecionales, así como los que deban serlo en virtud de disposición legal o reglamentaria expresa».

51. Articles 117.3 et 117.4 de la Constitution espagnole.

52. Real Decreto por el que se aprueba el Reglamento General de ingreso del personal al servicio de la Administración General del Estado y de provisión de puestos de trabajo y promoción profesional de los funcionarios civiles de la Administración General del Estado, nº 364/1995, 10 mars 1995, art. 56.2: «Las resoluciones de nombramiento se motivarán con referencia al cumplimiento por parte del candidato elegido de los requisitos y especificaciones exigidos en la convocatoria, y la competencia para proceder al mismo" ( La motivation des décisions de nomination doit mentionner la compétence de l'autorité investie de ce pouvoir ainsi que le respect, de la part du candidat sélectionné, des exigences et des spécificités propres à l'appel d'offre»).

53. STS, $7^{\mathrm{e}}$ sect., $\mathrm{n}^{\mathrm{o}} 5788 / 2006,29$ septembre $2006, \mathrm{FJ} 2$ : «El nombramiento para cargos de libre designación constituye un supuesto específico y singular dentro de la categoría general de los actos discrecionales, consistiendo la singularidad en que tales nombramientos se basan en la existencia de un motivo de confianza que sólo puede ser apreciado por la autoridad".

54. STS, $7^{\mathrm{e}}$ sect., $\mathrm{n}^{\circ}$ 8536/2002, 17 décembre 2002, FJ5: "La referencia a las condiciones subjetivas determinantes de la confianza que concurren en el designado para un cargo no son susceptibles de fiscalización en vía jurisdiccional».

55. Créée par le décret royal no 1/1977 (Boletín oficial del Estado, no 4, 5 janvier 1977, p. 172-174), l'Audiencia Nacional est une juridiction espagnole exclusivement compétente au niveau national pour un certain nombre de matières relevant du droit pénal, du droit administratif et du droit social.

56. STS, $1^{\mathrm{re}}$ sect., $\mathrm{n}^{\mathrm{o}}$ 3171/2006, 29 mai 2006, FJ 3 : «Por más que se trate en todo caso de aptitudes siempre referidas a idoneidad para el mejor desempeño del puesto y no a aspectos extraños y ajenos al mismo, como pudieran ser la empatía personal o la afinidad política». Commentaire: B. Porta Pego, « ¿El ocaso de la libre designación como sistema de provisión de algunos destinos en la carrera judicial ? La sentencia de la Sala Tercera del Tribunal Supremo del 29 de mayo de 2006, relativa al nombramiento del Presidente de la Sala de lo Penal de la Audiencia Nacional», Revista española de derecho administrativo, $\mathrm{n}^{\circ} 135,2007$, p. 591-612. 
à la question plus générale du rapport entre pouvoir discrétionnaire et contrôle juridictionnel de la motivation.

Or, le droit espagnol distingue le pouvoir discrétionnaire des «concepts juridiques indéterminés ${ }^{57}$. Ne figurant expressément pas dans la liste légale des cas soumis au devoir de motivation, ceux-ci peuvent ne pas être motivés. Mais la différence entre ces deux catégories repose sur un critère fragile et quelque peu déroutant. Dans le cadre du pouvoir discrétionnaire, l'administration pourrait choisir librement entre différentes options. En revanche, le contenu d'un concept juridique indéterminé est certes librement déterminé par l'administration, mais ne pourrait donner lieu qu'à une seule décision. Il en est ainsi, à titre d'exemple, de l'expression "candidat le plus adéquat» («candidato más adecuado») considérée comme un concept juridique indéterminé. L'administration serait ici libre de définir les contours de cette «adéquation» mais n'aurait alors, dans les faits, qu'un seul choix possible: celui de procéder au recrutement du candidat dont le profil est le plus adéquat. L'arrêt critiqué ${ }^{58}$ du 12 décembre $2000^{59}$ portant sur la nomination au poste de procureur du Tribunal suprême est une bonne illustration du flou entourant cette distinction. En l'espèce, le juge qualifie l'exigence d' «adéquation du profil» de «concept juridique indéterminé» et, sur ce fondement, ne censure pas le défaut de motivation.

«Artifice linguistique ${ }^{60}$, la distinction entre pouvoir discrétionnaire et «concept juridique indéterminé» repose sur une lecture littérale du texte de loi. Cette approche dichotomique est loin d'épouser une réalité plus complexe ${ }^{61}$. Il semble en tous les cas regrettable d'opposer ces deux notions au point de déduire que l'exigence de motivation, qui existe expressément pour l'une (pouvoir discrétionnaire) s'efface, a contrario, devant l'autre (concept juridique indéterminé). Il apparaît, à l'inverse, que l'exigence de motivation relative au pouvoir discrétionnaire devrait s'appliquer a fortiori en cas de «concept juridique indéterminé». L'on peut alors appeler à une interprétation «non pas littérale, mais téléologique ${ }^{62}$ du devoir légal de motivation.

N'est-ce pas en effet le pouvoir d'interprétation conceptuelle - et non l'existence d'une ou de plusieurs alternatives concrètes - qui doit avant tout guider le juge dans son contrôle? La même interrogation peut être soulevée à propos de la catégorie dénommée « discrétionnalité technique» («discrecionalidad técnica»). Son emploi justifierait, de manière tout aussi critiquable, la limitation du contrôle juridictionnel même si l'on note récemment une évolution vers un contrôle davantage poussé ${ }^{63}$.

Ainsi, le devoir de motivation est l'exemple topique de principes procéduraux indéterminables a priori. L'activisme du juge en la matière a amené à une réflexion plus profonde sur la nature de ce devoir. S'agit-il d'une pure formalité ou d'une exigence touchant au fond? Sur ce point, l'ordre juridique espagnol s'avère riche d'enseignements.

\section{Vers une approche substantielle de la motivation}

Depuis le passage à un État de droit social et démocratique, l'administration espagnole tire sa légitimité de son action efficace ${ }^{64}$. Renouvelant la conception classique du pouvoir discrétionnaire, certains auteurs ${ }^{65}$ contribuèrent ainsi à sa conception dynamique. Dans ce contexte, l'exigence de motivation n'a pas été diamétralement opposée aux motifs.

Cette approche s'explique sans doute en partie par la diversité des fondements constitutionnels du droit administratif espagnol ${ }^{66}$. Ainsi, la motivation des actes administratifs a très tôt était rattachée à des dispositions de la Constitution, ce qui exclut a priori de la considérer comme accessoire (A). Le détour par le droit espagnol permet ainsi de nourrir la réflexion sur les rapports entre le fond et la forme (B).

\section{A. Le rattachement du devoir de motivation à des principes et droits constitutionnels}

Même si le devoir de motivation des actes administratifs n'apparaît pas expressément dans le texte constitutionnel, de nombreux fondements sont invoqués à ce titre. Il en est ainsi de l'interdiction de l'arbitraire édictée, au même titre que la soumission de l'administration au principe de légalité, à l'article 9.3 de la Constitution ${ }^{67}$. De même, l'article 103.1 précité dispose que:

57. R. Bocanegra Sierra, A. Huergo Lora, «Un paso atrás en el control judicial de la discrecionalidad: su confusión con los conceptos jurídicos indeterminados y la dispensa del deber de motivar", Revista española de derecho administrativo, $\mathrm{n}^{\circ}$ 111, 2001, p. 405-428.

58. R. Bocanegra Sierra, A. Huergo Lora, «Un paso atrás...»; E. Desdentado Daroca, Discrecionalidad administrativa..., p. 89-91.

59. STS, $7^{\mathrm{e}}$ sect., $\mathrm{n}^{\circ}$ 9097/2000, 12 décembre 2000: à propos du décret royal nº 616/1999 du 16 avril 1999 procédant à la nomination du procureur du Tribunal suprême (fiscal del Tribunal supremo).

60. R. Bocanegra Sierra, A. Huergo Lora, «Un paso atrás... », p. 412.

61. N. MacCormick, Legal Reasoning and Legal Theory, Oxford, Clarendon Press, 1994, p. 103.

62. E. Desdentado Daroca, Discrecionalidad administrativa..., p. 91.

63. Ibid., p. 115-121.

64. L. Parejo Alfonso, Administrar y juzgar: dos funciones constitucionales distintas y complementarias, Madrid, Tecnos (Ciencias jurídicas), 1993, spéc. p. 96-98.

65. F. Sainz Moreno, Conceptos jurídicos, interpretación y discrecionalidad administrativa, Madrid, Civitas (Monografías), 1976.

66. Pour un commentaire systématique de la Constitution de 1978, voir Ó. Alzaga Villaamil, Comentario sistemático a la Constitución española de

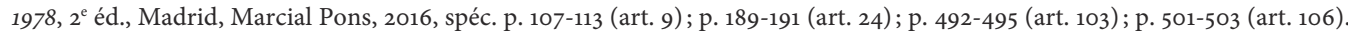

67. Art. 9.3 de la Constitution espagnole: «La Constitución garantiza el principio de legalidad, la jerarquía normativa, la publicidad de las normas, la irretroactividad de las disposiciones sancionadoras no favorables o restrictivas de derechos individuales, la seguridad jurídica, la responsabilidad y la interdicción de la arbitrariedad de los poderes públicos» («La Constitution garantit le principe de légalité, la hiérarchie des normes ainsi que leur publication, la non-rétroactivité des sanctions non favorables ou limitant des droits individuels, la sécurité juridique ainsi que la responsabilité 
L'administration publique satisfait l'intérêt général de manière objective et en respectant les principes d'efficacité, de hiérarchie, de décentralisation, de déconcentration et de coordination, et en se soumettant pleinement à la loi et au Droit ${ }^{68}$.

On peut aussi se référer à l'article 106.1 prévoyant l'existence d'un contrôle juridictionnel de l'action administrative ${ }^{69}$. À ce sujet, une comparaison intégrative peut être tentée avec la jurisprudence française. Ainsi, à titre d'exemple, dans un arrêt de $1948^{70}$ les juges du Palais-Royal ont considéré que le défaut de motivation en question n'avait «pas mis le Conseil d'État à même d'exercer le contrôle qui lui appartient $»^{71}$.

Le défaut de motivation peut aussi être envisagé comme une violation du droit constitutionnel à un recours juridictionnel effectif contenu à l'article 24.1 de la Constitution espagnole ${ }^{72}$. Ce dernier fondement contribue à subjectiver un devoir pensé de manière essentiellement objective. Ainsi que le rappelle le juge ${ }^{73}$, la motivation d'un acte administratif permet aussi au citoyen de se défendre contre l'administration lors d'un procès. Le défaut de motivation d'un tel acte serait alors une entrave au recours juridictionnel. Autrement dit, les éléments de motivation sont tout autant de moyens de défense du citoyen contre l'administration et leur défaut peut alors, selon les cas, constituer une violation du droit constitutionnel à un recours juridictionnel effectif. Ce raisonnement n'est pas sans rappeler le contexte d'élaboration de l'article 41 de la Charte européenne des droits fondamentaux relatif au droit à une bonne administration. Les rédacteurs de cet article se sont en effet ouvertement inspirés de l'article 6 de la Convention européenne des droits de l'homme relatif au droit à un procès équitable ${ }^{74}$.

Ainsi, en droit espagnol, le respect du devoir de motivation des actes administratifs ne se limite pas à la soumission à la loi ou à un Code de procédure administrative. Son manquement peut s'interpréter, selon les cas, comme une violation de la Constitution, ce qui lui confère une certaine substance.
On pourrait déduire de cette diversité de fondements une forme d'activisme de la part du juge constitutionnel. Toutefois, celui-ci se montre relativement prudent quant à sa compétence en la matière. Selon ses termes, la motivation des actes administratifs est une «question de légalité ordinaire ${ }^{75}$.

Toutefois, celui-ci est compétent en cas de sanctions administratives ou si le défaut de motivation de l'acte administratif est tel qu'il engendre la violation d'un droit fondamental. Mais le Tribunal constitutionnel se montre plutôt respectueux de la compétence principale du juge administratif. Cette parcimonie n'est pourtant pas exempte de critiques $^{76}$. En l'absence de motifs extériorisés dans la décision, le citoyen n'est-il pas ipso facto privé de moyens de défense (indefensión) ? Le défaut de motivation n'est-il pas, par lui-même, constitutif d'une violation du droit constitutionnel à un recours juridictionnel effectif?

Par contraste, la jurisprudence constitutionnelle espagnole s'avère particulièrement créative lorsqu'il s'agit de se prononcer sur le défaut de motivation des décisions juridictionnelles ${ }^{77}$. Ne serait-il pas alors opportun de s'inspirer de cette jurisprudence pour l'étendre à la motivation des actes administratifs?

Rejetant pour le moment cette éventualité, le Tribunal ne se déclare pas moins compétent s'agissant du défaut de motifs des actes administratifs. En effet, l'existence de motifs est interprétée comme une garantie contre l'arbitraire, dont l'interdiction fait l'objet de dispositions constitutionnelles, comme nous l'avons déjà évoqué. Notons toutefois que le juge constitutionnel conserve ici une certaine marge d'appréciation. En effet, de façon relativement fréquente, en cas de défaut de motivation, celui-ci se contente de renvoyer la question au juge administratif sans même vérifier l'existence de motif $^{78} \ldots$

Quoi qu'il en soit, l'étude de la motivation des actes administratifs espagnols passe inévitablement par une analyse de ses fondements constitutionnels, qui lui confèrent

et l'interdiction de l'arbitraire des pouvoirs publics»). Pour une illustration du rattachement de la motivation à l'interdiction de l'arbitraire: STC, $2^{\mathrm{e}}$ sect., $\mathrm{n}^{\mathrm{o}} 77 / 2000,27$ mars 2000, amparo $\mathrm{n}^{\circ} 3791 / 95$, FJ2.

68. Art. 103.1 de la Constitution espagnole: «La Administración Pública sirve con objetividad los intereses generales y actúa de acuerdo con los principios de eficacia, jerarquía, descentralización, desconcentración y coordinación, con sometimiento pleno a la ley y al Derecho ».

69. Art. 106.1 de la Constitution espagnole: «Los Tribunales controlan la potestad reglamentaria y la legalidad de la actuación administrativa, así como el sometimiento de ésta a los fines que la justifican» («Les tribunaux contrôlent le pouvoir réglementaire et la légalité de l'action administrative ainsi que la soumission de cette dernière aux finalités qui la justifient»).

70. CE, Ass., 27 février 1948, Société des Laboratoires du Bac et autres, $2^{\mathrm{e}}$ esp., $\mathrm{n}^{\text {os }}$ 80899, 80903, 80907, Recueil des arrêts du Conseil d'État, p. 101.

71. Ibid., cons. 4. Voir également dans ce sens: CE, sect., 27 janvier 1950, Sieur Billard, nº 99605, Recueil des arrêts du Conseil d’État, p. 58 ; Recueil Sirey, 1950, III, p. 41, concl. Letourneur.

72. Art. 24.1 de la Constitution espagnole: «Todas las personas tienen derecho a obtener la tutela efectiva de los jueces y tribunales en el ejercicio de sus derechos e intereses legítimos, sin que, en ningún caso, pueda producirse indefensión» ("Toute personne a droit à une protection juridictionnelle effective, dans l'exercice de ses droits et intérêts légitimes. En aucun cas ne pourra se produire un déni de justice»).

73. Pour exemple: STS, $5^{\mathrm{e}}$ sect., $\mathrm{n}^{\circ} 8178 / 2006,29$ novembre $2006, \mathrm{FJ} 4$ et 5.

74. J. Ziller, «Droit à une bonne administration", $\mathrm{n}^{\text {os }} 91-95$.

75. STC, $1^{\text {re }}$ sect., $\mathrm{n}^{\circ}$ 17/2009, 26 janvier 2009, $\mathrm{FJ}_{2}$ : «Cuestión de legalidad ordinaria que no alcanza dimensión constitucional ", à propos de la reconnaissance du parcours de recherche d'un professeur (sexenio).

76. E. Destentado Daroca, «La motivación de los actos administrativos y su control. Reflexiones críticas sobre las últimas orientaciones», Revista vasca de administración pública, $\mathrm{n}^{\circ} 84,2009$, p. 106-107.

77. Pour un exemple ancien, voir STC, $1^{\text {re }}$ sect., $n^{\circ} 5 / 1986,21$ janvier 1986, FJ2.

78. Pour exemple: STC, $1^{\text {re }}$ sect., $\mathrm{n}^{\circ}$ 17/2009, 26 janvier 2009. Notons toutefois que le Tribunal suprême lui-même se refuse aussi parfois à contrôler les motifs en l'absence de motivation: R. Bocanegra Sierra, A. Huergo Lora, «Un paso atrás... », p. 424. 
une dimension substantielle. Plus généralement, cet ordre juridique permet d'enrichir les réflexions sur les rapports entre «fond» et «forme».

\section{B. Un ordre juridique contribuant à la réflexion sur les rapports entre le fond et la forme}

La question des rapports entre le fond et la forme n'est pas uniquement conceptuelle. Si le devoir de motivation est considéré comme une exigence purement formelle, son non-respect pourra être compensé par une simple révision de l'acte, qui donnera lieu, le cas échéant, à l'indemnisation de l'intéressé sur le fondement du fonctionnement anormal de l'administration publique ${ }^{79}$. À l'inverse, s'il s'agit d'une exigence touchant au fond, l'annulation de l'acte sera quasi systématique. La jurisprudence espagnole n'est pas des plus prévisibles: les conséquences d'un défaut de motivation dépendent du cas concret et de la violation éventuelle d'une disposition constitutionnelle.

Or, la distinction entre le «formalisme essentiel» et le «formalisme subsidiaire» s'avère particulièrement hardie $^{80}$. À l'instar du droit français, une même formalité peut être substantielle ou accessoire suivant la gravité de l'illégalité dont elle est entachée. Il n'y a donc pas de formalité substantielle per se et la distinction figée entre la substance et la forme paraît peu opérante. La démarche suivie par le juge peut, de plus, paraître curieuse: c'est le dernier élément de la «chaîne», à savoir l'annulation, qui détermine le caractère substantiel de la formalité. Le lien d'imputation est donc inversé puisque l'opportunité de l'annulation dicte la qualification juridique des faits...

Indépendamment des conséquences pratiques du défaut de motivation, les termes de «fond » et de «forme» apparaissent parfois conjointement. Aussi le juge espagnol qualifie-t-il le devoir de motivation d' « exigence indispensable relevant non seulement de la forme, mais aussi du fond $»^{81}$. Ainsi que le rappelle le Tribunal constitutionnel, la motivation ne se réduit pas à une «courtoisie élémentaire ${ }^{82}$ puisque celle-ci permet de prendre connaissance du processus logique et juridique à l'origine de la décision.
Le vocable du Tribunal suprême est tout aussi incisif: la motivation n'est pas

[...] une formule conventionnelle ou purement rituelle, mais bien la spécification de la cause, c'est-à-dire de l'adéquation de l'acte par rapport à l'objectif poursuivi ${ }^{83}$.

Cette question a soulevé l'intérêt de la doctrine espagnole. Pour la majorité des auteurs ${ }^{84}$, à l'instar de l'approche traditionnelle française ${ }^{85}$, la motivation relève de la forme tandis que les motifs ont trait au fond de l'acte. Il serait même préjudiciable de qualifier la première d' «exigence de fond» car sa violation entraînerait des annulations systématiques, même en présence de motifs ${ }^{86}$. À l'inverse, selon une minorité de la doctrine espagnole ${ }^{87}$, le défaut de motivation convertit systématiquement l'acte administratif en acte arbitraire. De par cette inconstitutionnalité, l'annulation devrait alors être automatique, toute recherche de motifs s'avérant vaine. Certaines analyses plus mesurées ${ }^{88}$ considèrent que la motivation devrait être à présent considérée dans la plupart des cas comme une exigence de fond, en particulier lorsqu'aucun élément ne permet d'atteindre les motifs de la décision.

Cette volonté de ne pas réduire la motivation à la pure présence formelle de quelques «paragraphes d'explication » est peut-être à l'origine de la notion de «motivation par renvoi » («motivación por remisión ${ }^{89}$ ). Ici, la motivation d'un acte administratif peut consister en un simple renvoi à un rapport, un mémoire, un document figurant sur le dossier ou à une décision antérieure ${ }^{90}$. L'attention se porte sur le fond et, par conséquent, nul besoin de reproduire formellement la motivation déjà existante: l'administration doit simplement joindre les documents concernés à sa décision ${ }^{91}$. Une auteure ${ }^{92}$ a ainsi distingué la «motivation formelle-externe» («motivación formalexterna»), la «motivation formelle-interne» («motivación formal-interna») correspondant aux cas de «motivation par renvoi » et, enfin, les motifs («justificación o motivos»).

Suivant cette approche, la motivation est loin de constituer une simple formalité. Une expression employée par le Tribunal de justice de La Rioja dans un arrêt de 2016 illustre cette vision. En effet, en l'espèce, le juge qualifie

79. A. Huergo Lora, «La motivación de los actos administrativos y la aportación de nuevos motivos en el proceso contencioso-administrativo», Revista de administración pública, nº 145,1998, p. 106.

80. P. Gonod, «La simplification du droit par ordonnances», L'actualité juridique. Droit administratif, $\mathrm{n}^{\circ} 31,22$ septembre $2003, \mathrm{p} .1653$.

81. STSJ de Catalogne, $3^{\mathrm{e}}$ sect., $\mathrm{n}^{\circ}$ 11971/2009, 4 septembre 2009, FJ2: "un requisito no solo de forma pero también de fondo e indispensable ".

82. STC, $2^{\mathrm{e}}$ sect., $\mathrm{n}^{\circ} 26 / 1981,17$ juillet $1981, \mathrm{FJ} 14$ : «elemental cortesía ».

83. STS, $6^{\mathrm{e}}$ sect., $\mathrm{n}^{\mathrm{o}} 1623 / 2003,10$ mars $2003, \mathrm{FJ} 2$ : «[...] formula convencional ni meramente ritual, sino la especificación de la causa, esto es, la adecuación del acto al fin perseguido».

84. M. Sánchez Morón, Derecho administrativo..., p. 242 ; F. Garrido Falla, Tratado de derecho administrativo, $10^{\circ}$ éd., Madrid, Tecnos, 1987 , p. 436 sq

85. M. Waline, Droit administratif, $9^{\mathrm{e}}$ éd., Paris, Sirey (Traités Sirey), 1963, p. 458

86. E. Desdentado Daroca, Discrecionalidad administrativa..., p. 87.

87. T.-R. Fernández, De la arbitrariedad de la administración, $2^{\mathrm{e}}$ éd., Madrid, Civitas, 1997, p. 82 sq.; J. Igartua Salaverría, Discrecionalidad técnica, motivación y control jurisdiccional, $1^{\text {re }}$ éd., Madrid, Civitas (Cuadernos Civitas), 1998, p. 77.

88. J. Ponce Solé, Deber de buena administración y derecho al procedimiento administrativo debido, Valladolid, Lex Nova (Derecho público), 2001, p. 518-519.

89. Art. 88.6 de la loi $\mathrm{n}^{\circ} 39 / 2015$ du $1^{\mathrm{er}}$ octobre 2015 : «La aceptación de informes o dictámenes servirá de motivación a la resolución cuando se incorporen al texto de la misma".

90. STC, $1^{\text {re }}$ ch., $n^{\circ} 174 / 1987,3$ novembre 1987 , amparo $n^{\circ} 1072 / 1986$.

91. Pour exemple: STS, $4^{\text {e }}$ sect., $\mathrm{n}^{\circ}$ 116/2004, 19 janvier 2004

92. E. Desdentado Daroca, Discrecionalidad administrativa..., p. 97 et p. 102 sq. 
les organes consultatifs d' "auxiliaires à la formation de la volonté ${ }^{93}$ de l'administration. Leurs rapports doivent donc être publiés et joints à la décision car ceux-ci en constituent la motivation.

Garantie face au risque d'arbitraire, la motivation pourrait à ce titre être étendue aux raisons soutenant la décision au lieu d'être réduite à une simple description des motifs ${ }^{94}$. Sans adopter une telle position, on s'accordera à considérer que la tendance à reléguer les vices de forme au second plan ne paraît pas cohérente: la motivation conditionne l'examen des motifs. En effet, lorsqu'une décision est expressément motivée - que cette motivation soit obligatoire ou spontanée - et hormis les cas de substitution de motifs, le juge prend rarement en compte d'autres motifs que l'administration pourrait éventuellement lui fournir dans ses observations ${ }^{95}$.

La notion de motivation semble en réalité se situer "dans une zone d'ombre» entre le fond et la forme. Audelà de la description du droit positif espagnol, il apparaît ainsi utile de rappeler l'une des fonctions essentielles et pourtant peu usitées du droit comparé: celle consistant, par la confrontation de cultures juridiques différenciées, à déterminer le sens ou du moins l'approche vis-à-vis d'une notion ${ }^{96}$.

93. STSJ de La Rioja, $\mathrm{n}^{\circ}$ 10/2016, 14 janvier 2016, $\mathrm{FJ} 3$ : «Coadyuvantes a la formación de la voluntad del órgano activo ».

94. J. Igartua Salaverría, Discrecionalidad técnica..., p. 77.

95. Pour exemple: CE, sect., 23 juillet 1976, Ministre du Travail c. URSSAF du Jura, $\mathrm{n}^{\circ}$ 96526, Recueil Lebon, p. 362 ; Revue administrative, 1976, p. 607 , concl. Dondoux.

96. Sur une telle utilisation de la comparaison, voir L.-J. Constantinesco, Traité de droit comparé, t. II, La méthode comparative, Paris, LGDJ, 1974, n 118. 